\title{
ON AUTOMORPHISMS AND DERIVATIONS OF SIMPLE RINGS WITH MINIMUM CONDITION( $\left.{ }^{1}\right)$
}

\author{
BY \\ ANDRZEJ BIALYNICKI-BIRULA
}

I. Introduction. Let $A, B_{1}, B_{2}, C$ be fields such that $C \subset B_{1} \subset A$ and $C \subset B_{2} \subset A$, and suppose that $A$ is a finite and normal extension of $C$. Then it is known that the following conditions are equivalent:

(1) If $\phi_{i}$ is an isomorphism of $B_{i}$ into $A$ leaving the elements of $C$ fixed, $i=1$, 2, and $\phi_{1}\left|B_{1} \cap B_{2}=\phi_{2}\right| B_{1} \cap B_{2}$ then there exists an automorphismo of $A$ which is a common extension of $\phi_{1}$ and $\phi_{2}$.

(2) $B_{1}$ and $B_{2}$ are linearly disjoint over $B_{1} \cap B_{2}$.

$\left(2^{\prime}\right)\left(B_{1}, B_{2}\right) \approx B_{1} \otimes_{B 1 \cap B 2} B_{2}$.

$\left(2^{\prime \prime}\right)\left[\left(B_{1}, B_{2}\right): B_{1} \cap B_{2}\right]=\left[B_{1}: B_{1} \cap B_{2}\right] \cdot\left[B_{2}: B_{1} \cap B_{2}\right]$.

Moreover, if $B_{1}$ and $B_{2}$ are normal extensions of $C$ then the above conditions are satisfied.

The main purpose of this paper is to give some theorems of this type for division rings and simple rings with minimum condition. First we consider the case where $A, B_{1}, B_{2}, C$ are simple rings with minimum condition, $A$ is a Galois $\left({ }^{2}\right)$ extension of $C, B_{1}$ and $B_{2}$ are regular subrings $\left({ }^{3}\right)$ of $A$ containing $C$, and the center $Z(A)$ of $A$ is infinite. Then we prove (Theorem 1) that if (1) is satisfied then

(3) the centralizer of $B_{1} \cap B_{2}$ in $A$ is equal either to the centralizer of $B_{1}$, or to the centralizer of $B_{2}$.

Moreover, if $B_{1}$ and $B_{2}$ are normal extensions of $C$ then (1) is equivalent to (3) (Theorem 2), and $A=\left(B_{1}, B_{2}\right)$ and (1) imply (2") (Corollary 3).

Next we consider the case where $A, B_{1}, B_{2}, C$ are division rings, $A$ is a finite and normal extension of $C, B_{1}$ and $B_{2}$ are two division rings between $C$ and $A$ and the center of $A$ is infinite. Then we prove (Theorem 3) that (1) is equivalent to the conjunction of (2) and (3). As a consequence, we obtain that if $B_{1}$ and $B_{2}$ are normal over $C$ and $A=\left(B_{1}, B_{2}\right)$ then (1) implies $\left(2^{\prime}\right)$, where the sign $\approx$ occurring in condition $\left(2^{\prime}\right)$ is interpreted as an isomorphism of double $\left(B_{1} ; B_{2}\right)$-modules.

In the next part we consider the analogous question for derivations.

Finally, in the last part, we prove the following theorem $\left({ }^{4}\right)$ : Let $A, B, C$

Received by the editors June $16,1960$.

(1) This paper is a doctoral dissertation written at the University of California under the direction of Professor Gerhard P. Hochschild.

(2) See p. 471 for definition.

(3) See p. 471 for definition.

(4) For analogous result for division rings (without assumption that $A$ is a finite extension of $C$ ) see Theorem 1 p. 186 in [12]. 
be simple rings with minimum condition, let $C$ be a regular subring of $A$, and let $B$ be between $C$ and $A$. Moreover, suppose that the center of $A$ is infinite. If $B$ is carried into itself by every inner automorphism belonging to $G(A / C)\left({ }^{5}\right)$ then either $B$ contains the centralizer of $C$ in $A$ or every inner automorphism belonging to $G(A / C)$ leaves the elements of $B$ fixed. As a consequence of this result, we obtain a characterization of $A$-normal $\left(^{6}\right)$ extensions of $C$.

In conclusion, the author would like to express his warmest thanks to Professor Gerhard P. Hochschild for his advice and encouragement in the preparation of this paper.

2. Throughout this paper, $P, R$ will denote rings with an identity element. By a subring of a ring with an identity element we mean a subring that contains this identity element.

Let $C$ be a simple subring of $R$ with minimum condition. Then we shall often consider $R$ as a left $C$-module. It is known $\left({ }^{7}\right)$ that this $C$-module is free and has a well defined dimension over $C$. The dimension will be denoted by $[R: C]$. We shall say that $R$ is a finite extension of $C$ if this $C$-module is finite dimensional, i.e., if $[R: C]<\infty$.

If $a \in R$ then $a_{r}$ denotes the mapping of $R$ into $R$ defined by the equation $a_{r}(x)=x a$, for every $x \in R$. If $S$ is a subset of $R$ then $S_{r}$ denotes the set of all mappings $a_{r}$, for $a \in S$. Similarly, $a_{l}$ denotes the mapping defined by $a_{l}(x)$ $=a x$, for every $x \in R$, and $S_{l}$, where $S \subset R$, denotes the set of all mappings $a_{l}$, where $a \in S$. If $S_{1}$ and $S_{2}$ are two subsets of $R$ then $S_{1} \cdot S_{2}$ denotes the set of all elements of the form $s_{1} \cdot s_{2}$, where $s_{i} \in S_{i}$, for $i=1,2$, and $\left(S_{1}, S_{2}\right.$ denotes the subring of $R$ generated by $S_{1} \cup S_{2}$ ).

$R^{*}$ denotes the set of all units of $R . Z(R, C)$ denotes the centralizer of $C$ in $R$. The center of $R$ will be denoted by $Z(R)$. If $a$ is a unit of $R$ then $I_{a}$ denotes the automorphism of $R$ defined by the equation $I_{a}(x)=a x a^{-1}$, for every $x \in R . G(R / C)$ denotes the group of all automorphisms of $R$ leaving the elements of $C$ fixed. Let $G_{1}$ be a subgroup of $G(R / C)$. Then $R^{G_{1}}$ denotes the set of all elements $a \in R$ such that, for every $\phi \in G_{1}, \phi(a)=a . G_{1}^{0}$ denotes the subgroup of $G_{1}$ consisting of all inner automorphisms belonging to $G_{1} . R\left(G_{1}\right)$ denotes the subring of $R$ generated by the invertible elements $a \in R$ such that $I_{a} \in G_{1}$. We say that $G_{1}$ is complete if, for every invertible element $a \in R\left(G_{1}\right)$, $I_{n} \in G_{1} . R$ is called a normal extension of $C$ if $C=R^{G(A / C)}$.

In the sequel, letters $A, B_{1}, B_{2}, C, D$ will denote simple rings with minimum condition.

(i) Let $C$ be a subring of $A$. Let $\sigma_{1}, \cdots, \sigma_{n}$ be elements of $G(A / C)$ such that $G(A / C)^{0} \sigma_{s} \neq G(A / C)^{0} \sigma_{t}$, when $s \neq t$, and let $z_{1}, \cdots, z_{m}$ be elements of $A$ that are linearly independent over $Z(A)$. Then $\left(z_{1}\right)_{l} \sigma_{1}, \cdots,\left(z_{i}\right)_{l} \sigma_{j}, \cdots$, $\left(z_{m}\right)_{l} \sigma_{n}$ are linearly independent over $A_{r}$.

(5) See definition below.

${ }^{(6)}$ See p. 471 for definition.

( ${ }^{7}$ See e.g. p. 53 in [3]. 
Proof. Suppose that $\left(z_{1}\right)_{l} \sigma_{1}, \cdots,\left(z_{i}\right)_{l} \sigma_{j}, \cdots,\left(z_{m}\right)_{l} \sigma_{n}$ are linearly dependent over $A_{r}$. Let $k+1$ be the minimal number of nonzero coefficients in the nontrivial $A_{r}$-linear relations among the $\left(z_{i}\right)_{i} \sigma_{j}$. Then there exist $a_{i j} \in A$, $i=1, \cdots, m, j=1, \cdots, n$, such that $\sum_{i=1}^{m} \sum_{j=1}^{n}\left(a_{i j}\right)_{r}\left(z_{i}\right)_{i} \sigma_{j}=0$, where $a_{i j}=0$ for all but $k+1$ pairs $(i, j)$. We may assume that $a_{11} \neq 0$. Since $A$ is a simple ring, the two-sided ideal $A a_{11} A$ coincides with $A$ and there are elements $x_{p}, y_{p}, p=1, \cdots, q, x_{p}, y_{p} \in A$ such that $\sum_{p=1}^{q} x_{p} a_{11} y_{p}=1$, whence $\sum_{p=1}^{g}\left(y_{p}\right)_{r}\left(a_{11}\right)_{r}\left(x_{p}\right)_{r}=1_{r}$. Multiplying the relation $\sum_{i, j}\left(a_{i j}\right)_{r}\left(z_{i}\right)_{l} \sigma_{j}=0$ by $\left(y_{p}\right)_{r}$ from the left and by $\left(\sigma_{1}^{-1}\left(x_{p}\right)\right)_{r}$ from the right and summing for $p=1, \cdots, q$ we obtain the relation $\sum_{i, j}\left(b_{i j}\right)_{r}\left(z_{i}\right)_{l} \sigma_{j}=0$, where $b_{i j} \in A$, $b_{11}=1$ and $b_{i j} \neq 0$ for exactly $k+1$ pairs $(i, j)$. Let $a \in A$. Then we have $\sum_{i, j} a_{r}\left(b_{i j}\right)_{r}\left(z_{i}\right)_{l} \sigma_{j}=0$ and $\sum_{i, j}\left(b_{i j}\right)_{r}\left(z_{i}\right)_{l} \sigma_{j}\left(\sigma_{1}^{-1}(a)\right)_{r}=0$, i.e.,

$$
\sum_{i, j}\left(b_{i j}\right)_{r}\left(\sigma_{j} \sigma_{1}^{-1}(a)\right)_{r}\left(z_{i}\right)_{l} \sigma_{j}=0 \text {. }
$$

Subtracting this from the previous relation we obtain $\sum_{i, j}\left(c_{i j}\right)_{r}\left(z_{i}\right)_{i} \sigma_{j}=0$, where $c_{i j}=b_{i j} a-\sigma_{j} \sigma_{1}^{-1}(a) b_{i j}$ and the number of pairs $(i, j)$ for which $c_{i j} \neq 0$ is less than $k+1$ since $c_{11}=0$. Hence, we obtain, by the minimality of $k+1$, that $c_{i j}=0$, for all $i=1, \cdots, m, j=1, \cdots, n$. Thus $b_{i j} a-\sigma_{j} \sigma_{1}^{-1}(a) b_{i j}=0$. This shows that $b_{i j} A=A b_{i j}$, and, since $A$ is simple, we may conclude that each $b_{i j}$ is either 0 or a unit. Therefore, if $b_{i j} \neq 0$ then $\sigma_{j} \sigma_{1}^{-1}=I_{b_{i j}}$ and so $j=1$, since $G(A / C){ }^{0} \sigma_{s} \neq G(A / C)^{0} \sigma_{t}$, when $s \neq t$. Hence we may label the $z_{i}$ so that there are nonzero elements $b_{i 1} \in A, i=1, \cdots, k+1$, such that $\sum_{i=1}^{k+1}\left(b_{i 1}\right)_{r}\left(z_{i}\right)_{l} \sigma_{1}=0$ and $b_{11}=1$, i.e., $\left(z_{1}\right)_{l}+\sum_{l=2}^{k+1}\left(b_{i 1}\right)_{r}\left(z_{i}\right)_{l}=0$. Now let $a \in A^{*}$. Then $a_{r}^{-1}\left(z_{1}\right)_{l} a_{r}$ $+\sum_{i=2}^{k+1} a_{r}^{-1}\left(b_{i 1}\right)_{r} a_{r}\left(z_{i}\right)_{l}=0$ and so $\left(z_{1}\right)_{l}+\sum_{i=2}^{k+1}\left(a b_{i 1} a^{-1}\right)_{r}\left(z_{i}\right)_{l}=0$. Subtracting the last relation from $\left(z_{1}\right)_{l}+\sum_{i=2}^{k+1}\left(b_{i 1}\right)_{r}\left(z_{i}\right)_{l}=0$, we obtain

$$
\sum_{i=2}^{k+1}\left(b_{i 1}-a b_{i 1} a^{-1}\right)_{r}\left(z_{i}\right)_{l}=0 \text {. }
$$

By the minimality of $k+1$, this implies $b_{i 1}=a b_{i 1} a^{-1}$. Hence $b_{i 1} \in Z(A)$, because $A^{*}$ generates $A$. Thereforé, $\left(z_{1}\right)_{l}+\sum_{i=2}^{k+1}\left(b_{i 1}\right)_{l}\left(z_{i}\right)_{l}=0$ and so $z_{1}+\sum_{i=2}^{k+1} b_{i 1} z_{i}=0$. This gives a contradiction, because we have supposed that the $z_{i}$ are linearly independent over $Z(A)$. Thus the theorem is proved $\left({ }^{8}\right)$.

The following is well known $\left({ }^{9}\right)$ :

(ii) Let $C$ be a subring of $A$ and let $[A ; C]<\infty$. Let $E(A ; C)$ be the ring of all endomorphisms of the $C$-module $A$. Then $\left[E(A ; C): A_{\mathrm{r}}\right]=[A: C]$.

Combining (i) and (ii) we obtain

(iii) Let $C$ be a subring of $A$ and let $[A: C]<\infty$. Then $[Z(A, C): Z(A)]$ - $\left[G(A / C): G(A / C)^{0}\right] \leqq[A: C]<\infty$. In particular, $[Z(A, C): Z(A)]<\infty$.

REMARK. We shall prove later $\left({ }^{10}\right)$ that if a ring $R$ with the identity element

( $\left.{ }^{8}\right)$ The proof is patterned after a proof of a theorem of G. Hochschild.

(9) See e.g. p. 55 in [3].

(10) See Lemma 2. 
1 contains an infinite field $F$ such that $[R: F]<\infty$ then every element of $R$ can be represented as a sum of units. Hence it follows from (iii) that if $Z(A)$ is infinite than $Z(A, C)=R(G(A / C))$.

Let $C$ be a subring of $A$. Then $C$ is called a regular subring of $A$ if $C$ is simple with minimum condition, $[A: C]<\infty$ and $Z(A, C)$ is simple.

A subgroup $G_{1}$ of $G(A / C)$ is said to be regular if: (1) $G_{1}$ is complete, (2) $\left[G_{1}: G_{1}^{0}\right]<\infty$ and (3) $R\left(G_{1}\right)$ is simple and $\left[R\left(G_{1}\right): Z(A)\right]<\infty$.

$A$ is called a Galois extension of a subring $P$ if $A^{G(A / P)}=P$ and $G(A / P)$ is regular.

(iv)(11) If $G_{1}$ is a regular group of automorphisms of $A$ then $A^{G_{1}}$ is a regular subring of $A$, and $\left[A: A^{G_{1}}\right]=\left[G_{1}: G_{1}^{0}\right] \cdot\left[R\left(G_{1}\right): Z(A)\right]$. Hence if $A$ is a Galois extension of $P$ then $P$ is a regular subring of $A$.

REMARK. It follows from (iv) that if $[Z(A, C): Z(A)] \cdot\left[G(A / C): G(A / C)^{0}\right]$ $=[A: C]$ and $G(A / C)$ is regular then $C=A^{G(A / C)}$ and hence is a regular subring of $A$. One can also prove that if $[A: C)=[Z(A, C): Z(A)]$ - $\left[G(A / C): G(A / C)^{0}\right]$, which is equivalent to the assumption that $A$ is a strongly normal $\left({ }^{12}\right)$ extension of $C$, then $C$ is "close" to being regular; namely, $Z(A, C)$ is then a direct sum of mutually isomorphic simple rings.

Let $C \subset B \subset A$ be successive finite extensions of $C$. If $\phi \in G(A / C)$ then $\phi \mid B$ denotes the restriction of $\phi$ to $B$. If $G_{1}$ is a subgroup of $G(A / C)$ then $G_{1} \mid B$ denotes the set of all elements of the form $\phi \mid B$ where $\phi \in G_{1}$. Let $B$ be a regular subring of $A$. Then $G(B, A / C)$ denotes the set of all isomorphisms $\phi$ of $B$ into $A$ leaving the elements of $C$ fixed and such that $\phi(B)$ is a regular subring of $A$. It is easy to see that if $\psi \in G(A / C)$ then $\psi \mid B \in G(B, A / C)$.

$B$ is called an $A$-normal extension of $C$ if for every $\phi \in G(A / C) \phi(B)=B$.

Let $B_{1}$ and $B_{2}$ be extensions of $C$ and let $A$ be an extension of $B_{1}$ and $B_{2}$. If $\phi_{1} \in G\left(B_{1}, A / C\right), \phi_{2} \in G\left(B_{2}, A / C\right)$ then an element $\phi \in G(A / C)$ is called an extension of the pair $\left(\phi_{1}, \phi_{2}\right)$ if $\phi \mid B_{1}=\phi_{1}$ and $\phi \mid B_{2}=\phi_{2}$. The pair $\left(\phi_{1}, \phi_{2}\right)$ is called compatible if $\phi_{1}\left|B_{1} \cap B_{2}=\phi_{2}\right| B_{1} \cap B_{2}$.

In the sequel we shall use the following theorems:

(v) (13) Fundamental Theorem of Galois Theory. Let $A$ be a Galois extension of $C$. Let $\mathfrak{A}$ be the collection of all regular subgroups of $G(A / C)$ and let $\mathfrak{B}$ be the collection of all regular subrings of $A$ containing $C$. Then there exists the usual 1-1 correspondence between $\mathfrak{A}$ and $\mathfrak{B}$. In the direction from $\mathfrak{A}$ to $\mathfrak{B}$, we have $G_{1} \rightarrow A^{G_{1}} ;$ in the other direction, we have $B \rightarrow G(A / B)$.

(vi) ( $\left.{ }^{14}\right)$ Let $A$ be a Galois extension of $C$ and let $B$ be a regular subring between $C$ and $A$. Then every isomorphism $\phi \in G(B, A / C)$ has an extension $\psi \in G(A / C)$.

(11) See Theorem 1, p. 282 in [14].

(12) See p. 277 in [14] for definition.

(13) See Theorem 5 , p. 285 , in [14].

( $\left.{ }^{14}\right)$ See Theorem 6, p. 285, in [14]. 
Lemma 1. Let $A$ be a Galois extension of $C$ and let $B_{1}, B_{2}$ be regular subrings between $C$ and $A$. The following three conditions are equivalent:

(a) $G\left(A / B_{2}\right) \mid B_{1}=G\left(B_{1}, A / B_{1} \cap B_{2}\right)$.

(b) every compatible pair $\left(\phi_{1}, \phi_{2}\right)$, where $\phi_{i} \in G\left(B_{i}, A / C\right)$, for $i=1,2$, has an extension $\phi \in G(A / C)$.

(c) $G\left(A / B_{1} \cap B_{2}\right)=G\left(A / B_{2}\right) \cdot G\left(A / B_{1}\right)$.

Proof. (a) $\Rightarrow$ (b). Let us take $\phi_{1}$ and $\phi_{2}$ as in (b). It follows from (vi) that there exists $\psi_{2} \in G(A / C)$ such that $\psi_{2} \mid B_{2}=\phi_{2}$. Let us put $\gamma=\psi_{2}^{-1} \phi_{1}$. Then $\gamma \in G\left(B_{1}, A / B_{1} \cap B_{2}\right)$ and it follows from (a) that there exists $\psi \in G\left(A / B_{2}\right)$ such that $\psi \mid B_{1}=\gamma$. Now $\psi_{2} \psi\left|B_{1}=\psi_{2} \psi_{2}^{-1} \phi_{1}\right| B_{1}=\phi_{1} \mid B_{1}$ and $\psi_{2} \psi\left|B_{2}=\psi_{2}\right| B_{2}=\phi_{2}$. Hence $\psi_{2} \psi$ is an extension of $\left(\phi_{1}, \phi_{2}\right)$.

(b) $\Rightarrow$ (c). Let $\phi \in G\left(A / B_{1} \cap B_{2}\right)$. It follows from (b) that there exists an automorphism $\psi \in G\left(A / B_{1} \cap B_{2}\right)$ such that $\psi\left|B_{2}=\phi\right| B_{2}$ and $\psi \mid B_{1}$ is the identity automorphism of $B_{1}$. Now $\phi=\left(\phi \psi^{-1}\right) \psi, \phi \psi^{-1} \in G\left(A / B_{2}\right), \psi \in G\left(A / B_{1}\right)$. Thus $\phi \in G\left(A / B_{2}\right) \cdot G\left(A / B_{1}\right)$.

(c) $\Rightarrow$ (a). It follows from (c) that $G\left(A / B_{1} \cap B_{2}\right)\left|B_{1}=G\left(A / B_{2}\right) G\left(A / B_{1}\right)\right| B_{1}$ $=G\left(A / B_{2}\right) \mid B_{1}$. But by (vi) $G\left(A / B_{1} \cap B_{2}\right) \mid B_{1}=G\left(B_{1}, A / B_{1} \cap B_{2}\right)$, whence $G\left(A / B_{2}\right) \mid B_{1}=G\left(B_{1}, A / B_{1} \cap B_{2}\right)$.

The following corollary is an immediate consequence of the lemma:

Corollary 1. Let $A, B_{1}, B_{2}, C$ be as in Lemma 1. If $G\left(A / B_{2}\right) \mid B_{1}$ $=G\left(B_{1}, A / B_{1} \cap B_{2}\right)$ then $G\left(A / B_{1}\right) \mid B_{2}=G\left(B_{2}, A / B_{1} \cap B_{2}\right)$.

Let $V$ be a finite dimensional vector space over a field $F$. If $S$ is a subset of $V$ then $\bar{S}$ denotes the smallest algebraic subset of $V$ containing $S$.

LEMMA 2. Let $R$ be a ring with an identity element, let $F$ be an infinite field contained in $R$ and suppose that $[R: F]<\infty$. Let us consider $R$ as a left vector space over $F$. Then $\left(R^{*}\right)^{-}=R$.

Proof. Let $d$ be the function on $R$ defined by $d(a)=\operatorname{det}\left(a_{r}\right)$. Then $d$ is evidently a polynomial function on $R$, and the elements of $R^{*}$ are precisely the elements $a$ such that $d(a) \neq 0$. Now let $p$ be a polynomial function vanishing on $R^{*}$. Then $d p=0$. Since $d \neq 0$, this gives $p=0$. Thus the ideal associated with $R^{*}$ is the 0 -ideal, whence $\left(R^{*}\right)^{-}=R$.

Lemma 3. Let $R$ be a finite dimensional algebra over an infinite field $F$. Let $\left\{V_{j}, W_{j}\right\}_{j=1, \cdots, n}$ be a family of pairs of $F$-vector spaces contained on $R$. If $R=U_{j=1}^{n}\left(V_{j} W_{j}\right)^{-}$then there exists an index $j_{0}$, such that either $\operatorname{dim} V_{j_{0}}$ $>2^{-1} \operatorname{dim}_{F} R$ or $\operatorname{dim} W_{j_{0}}>2^{-1} \operatorname{dim}_{F} R$.

Proof. Let $T_{j}=\left(V_{j} W_{j}\right)^{-}, p_{j}=\operatorname{dim} V_{j}, q_{j}=\operatorname{dim} W_{j}$. Then $\operatorname{dim} T_{j} \leqq p_{j}+q_{j}$ -1. Indeed, let $M_{j}$ be a $\left(p_{j}-1\right)$-dimensional $F$-vector subspace of $V_{j}$, let $a_{j} \in V_{j}, a_{j} \notin M_{j}$ and suppose that $a_{j}+M_{j}$ is the set of all elements of the form $a_{j}+m$, where $m \in M_{j}$. Then it is easy to see that $V_{j} \cdot W_{j}=\left(a_{j}+M_{j}\right) \cdot W_{j}$ $\cup M_{j} \cdot W_{j}$. Moreover $\left(a_{j}+M_{j}\right) \times W_{j}$ and $M_{j} \times W_{j}$ are $\left(p_{j}-1+q_{j}\right)$-dimensional 
algebraic subsets of $V_{j} \times W_{j}$, and the mapping $f_{j}$ from $V_{j} \times W_{j}$ into $R$ defined by the equality $f_{j}(x, y)=x y$, for every $x \in V_{j}, y \in W_{j}$, is a polynomial mapping. Hence $\operatorname{dim} T_{j_{n}} \leqq p_{j}+q_{j}-1$.

Now let us suppose that $R=\bigcup_{j=1}^{n}\left(V_{j} W_{j}\right)^{-}=\bigcup_{j=1}^{n} T_{j}$. Then there exists an index $j_{0}$ such that $T_{j_{0}}=R$, because $R$ is irreducible. Hence $\operatorname{dim}_{F} R=\operatorname{dim} T_{j_{0}}$ $\leqq p_{j_{0}}+q_{j_{0}}-1$. Let us suppose that $p_{j_{0}} \leqq q_{j_{0}}$. Then $\operatorname{dim}_{F} R \leqq 2 q_{j_{0}}-1<2 q_{j_{0}}$, hence $q_{j_{0}}>2^{-1} \operatorname{dim}_{F} R$ and the lemma is proved.

Lemma 4. Let $R$ be a finite dimensional algebra with the identity 1 over an infinite field $F$. Let $\left\{C_{j}, D_{j}\right\}_{j=1, \cdots, n},\left\{d_{j}\right\}_{j=1, \cdots, n}$ be a family of pairs of simple rings with minimum condition between $F$ and $R$, and a subset of $R$, respectively. If $R^{*}=\bigcup_{j=1}^{n} C_{j}^{*} D_{j}^{*} d_{j}$ then there exists an index $j_{0}$, such that either $R=C_{j_{0}}$ or $R=D_{j_{0}}$.

Proof. Let us suppose that $R^{*}=\bigcup_{j=1}^{n} C_{j}^{*} D_{j}^{*} d_{j}$. Then, by Lemma 2, $R=\left(R^{*}\right)^{-}=\bigcup_{j=1}^{n}\left(C_{j}^{*} D_{j}^{*} d_{j}\right)^{-} \subset \bigcup_{j=1}^{n}\left(C_{j} D_{j} d_{j}\right)^{-}$. But $C_{j}$ and $D_{j} d_{j}$ are $F$-vector subspaces of $R$. Therefore, it follows from Lemma 3 that there exists an index $j_{0}$ such that either $\left[C_{j_{0}}: F\right]=\operatorname{dim}_{F} C_{j_{0}}>2^{-1} \operatorname{dim}_{F} R=2^{-1}[R: F]$ or $\operatorname{dim}_{F} D_{j_{0}} \cdot d_{j_{0}}$ $>2^{-1}[R: F]$. Hence either $\left[C_{j_{0}}: F\right]>2^{-1}[R: F]$ or $\left[D_{j_{0}}: F\right]>2^{-1}[R: F]$. But $[R: F]$ is divisible by $\left[C_{j_{0}}: F\right]$ and $\left[D_{j_{0}}: F\right]$. Therefore we get that either $[R: F]=\left[C_{j_{0}}: F\right]$ or $[R: F]=\left[D_{j_{0}}: F\right]$. If $[R: F]=\left[C_{j_{0}}: F\right]$ then $R=C_{j_{0}}$; if $[R: F]=\left[D_{j_{0}}: F\right]$ then $R=D_{j_{0}}$. This completes the proof of the lemma.

Theorem 1. Let $A$ be a Galois extension of $C$. Let $B_{1}, B_{2}$ be regular subrings between $C$ and $A$ and suppose that $Z(A)$ is infinite. If every compatible pair $\left(\phi_{1}, \phi_{2}\right)$, where $\phi_{i} \in G\left(B_{i}, A / C\right)$, for $i=1,2$, has an extension $\phi \in G(A / C)$ then either $Z\left(A, B_{1} \cap B_{2}\right)=Z\left(A, B_{1}\right)$ or $Z\left(A, B_{1} \cap B_{2}\right)=Z\left(A, B_{2}\right)$.

Proof. Let us suppose that every compatible pair $\left(\phi_{1}, \phi_{2}\right)$ where $\phi_{i} \in G\left(B_{i}, A / C\right)$, for $i=1,2$, has an extension $\phi \in G(A / C)$. Then it follows from Lemma 1 that $G\left(A / B_{1} \cap B_{2}\right)=G\left(A / B_{2}\right) \cdot G\left(A / B_{1}\right)$. Let $\left\{\phi_{1}^{i}, \cdots, \phi_{m_{i}}^{i}\right\}$, $i=1,2$, be a set of representatives of the cosets of the subgroup $G\left(A / B_{i}\right)^{0}$ in $G\left(A / B_{i}\right)$. Then every automorphism $\psi_{i} \in G\left(A / B_{i}\right), i=1,2$, can be represented in the form $I_{c_{i}} \phi_{j_{i}}^{i}$, where $1 \leqq j_{i} \leqq m_{i}$, and $c_{i} \in Z\left(A, B_{i}\right)^{*}$. For every $j$, $1 \leqq j \leqq m_{2}$, there exists at most one integer $k, 1 \leqq k \leqq m_{2}$, such that $\phi_{j}^{2} \phi_{k}^{1}$ is an inner automorphism. Indeed, if $\phi_{j}^{2} \phi_{k_{1}}^{1}$ and $\phi_{j}^{2} \phi_{k_{2}}^{1}$ are inner then also $\left(\phi_{j}^{2} \phi_{k_{1}}^{1}\right)^{-1} \phi_{j}^{2} \phi_{k_{2}}^{1}$ is inner. But $\left(\phi_{j}^{2} \phi_{k_{1}}^{1}\right)^{-1} \phi_{j}^{2} \phi_{k_{2}}^{1}=\left(\phi_{k_{1}}^{1}\right)^{-1} \phi_{k_{2}}^{1}$, hence $k_{1}=k_{2}$. Therefore we may suppose that the only inner automorphisms of the form $\phi_{j}^{2} \phi_{k}^{1}$ are the $\phi_{j}^{2} \phi_{j}^{1}$, with $1 \leqq j \leqq n$. For each such $j$ choose $c_{j} \in Z\left(A, B_{1} \cap B_{2}\right)^{*}$ such that $\phi_{j}^{2} \phi_{j}^{1}=I_{c j}$.

Let $a \in Z\left(A, B_{1} \cap B_{2}\right)^{*}$, then $I_{a}$ can be represented in the form $\phi_{2} \phi_{1}$, where $\phi_{i} \in G\left(A / B_{i}\right)$, for $i=1,2$, and hence in the form $I_{b} \phi_{j}^{2} I_{c} \phi_{k}^{1}$, where $b \in Z\left(A, B_{2}\right)^{*}, c \in Z\left(A, B_{1}\right)^{*}, 1 \leqq j \leqq m_{2}$ and $1 \leqq k \leqq m_{1}$. Therefore $I_{a}=I_{b} I_{\phi_{j}^{2}(c)} \phi_{j}^{2} \phi_{\mathbf{k}}^{1}$ and $\phi_{j}^{2} \phi_{k}^{1}$ is inner. Hence we get $j=k$ and $\phi_{j}^{2} \phi_{k}^{1}=I_{c_{j}}$. Thus every element $a \in Z\left(A, B_{1} \cap B_{2}\right)^{*}$ can be represented in the form $b d c_{j}$, where $b \in Z\left(A, B_{2}\right)^{*}, d \in \phi_{j}^{2}\left(Z\left(A, B_{1}\right)^{*}\right)$ and $1 \leqq j \leqq n$. On the other hand, $Z\left(A, B_{2}\right)^{*}$ 
- $\phi_{j}^{2}\left(Z\left(A, B_{1}\right)^{*}\right) \cdot c_{j} \subset Z\left(A, B_{1} \cap B_{2}\right)^{*}$, for every $1 \leqq j \leqq n$. Hence $Z\left(A, B_{1} \cap B_{2}\right)^{*}$ $=\bigcup_{j-1}^{n} Z\left(A, B_{2}\right)^{*} \cdot \phi_{j}^{2}\left(Z\left(A, B_{1}\right)^{*}\right) \cdot c_{j}$. Now $Z\left(A, B_{1} \cap B_{2}\right)$ is a finite extension of the infinite field $Z(A)$ and, for every $\phi \in G\left(A / B_{1} \cap B_{2}\right)$ and $i=1,2, \phi\left(Z\left(A, B_{i}\right)\right)$ is a simple ring with minimum condition containing $Z(A)$. Hence it follows from Lemma 4 that either $Z\left(A, B_{1} \cap B_{2}\right)=Z\left(A, B_{2}\right)$ or $Z\left(A, B_{1} \cap B_{2}\right)=\phi_{j}^{2}\left(Z\left(A, B_{1}\right)\right)$ for some $j$. Since $\phi_{j}^{2} \in G\left(A / B_{1} \cap B_{2}\right)$, the second equality implies that $Z\left(A, B_{1} \cap B_{2}\right)=Z\left(A, B_{1}\right)$. Therefore we conclude that either $Z\left(A, B_{1} \cap B_{2}\right)=Z\left(A, B_{2}\right)$ or $Z\left(A, B_{1} \cap B_{2}\right)=Z\left(A, B_{1}\right)$ and the theorem is proved.

Corollary 2. Let $A$ be a Galois extension of $C$. Let $B_{1}, B_{2}$ be two regular subrings between $C$ and $A$ and suppose that $Z(A)$ is infinite. If every compatible pair $\left(\phi_{1}, \phi_{2}\right)$, where $\phi_{i} \in G\left(B_{i}, A / C\right)$, for $i=1,2$, has an extension $\phi \in G(A / C)$ then either $G\left(B_{1} / B_{1} \cap B_{2}\right)$ or $G\left(B_{2} / B_{1} \cap B_{2}\right)$ contains no nontrivial inner automorphisms.

Proof. It follows from Theorem 1 that if the assumption of the corollary is satisfied then either $Z\left(A, B_{1} \cap B_{2}\right)=Z\left(A, B_{1}\right)$ or $Z\left(A, B_{1} \cap B_{2}\right)=Z\left(A, B_{2}\right)$. If $Z\left(A, B_{1} \cap B_{2}\right)=Z\left(A, B_{1}\right)$ then $Z\left(B_{1}, B_{1} \cap B_{2}\right)=Z\left(B_{1}\right)$ and therefore $G\left(B_{1} / B_{1} \cap B_{2}\right)^{0}$ is trivial. Similarly, if $Z\left(A, B_{1} \cap B_{2}\right)=Z\left(A, B_{2}\right)$ then $G\left(B_{2} / B_{1} \cap B_{2}\right)^{0}$ is trivial.

Lemma 5. Let $C \subset P \subset B \subset A$ be successive extensions of $C$ such that $A$ is a Galois extension of $C, B$ is a regular subring of $A$ and $B$ is a normal extension of $P$. If $B \subset A^{G(A / P)^{0}}$ then $P$ is a regular subring of $A, B$ is an $A$-normal extension of $P$, and $G(B / P)$ is finite.

Proof. Let us suppose that $B \subset A^{G(A / P)^{0}}$. Then $G(B / P)$ contains no nontrivial inner automorphism. Hence $\operatorname{card}(G(B / P))=\left[G(B / C): G(B / C)^{0}\right]$. But it follows from (iii) that $\left[G(B / C): G(B / C)^{0}\right] \leqq[B: C]$. Thus $G(B / P)$ is finite, since $[B: C] \leqq[A: C]<\infty$. Hence $G(B / P)$ is regular, and it follows from (iv) that $P$ is a regular subring of $B$. Since $B \subset A^{G(A / P)^{0}}$, we have $Z(A, P)=Z(A, B)$, so that $Z(A, P)$ is simple. As a regular subring of $B, P$ is simple with minimum condition. Also $[A: P] \leqq[A: C]<\infty$, and we conclude that $P$ is a regular subring of $A$.

Now let $D=A^{G(A / P)^{0}}$. Then $D$ is an $A$-normal extension of $P$. Therefore, in order to prove that $B$ is an $A$-normal extension of $P$, it suffices to show that $B$ is a $D$-normal extension of $P . D$ is a regular subring of A because $D=A^{G(A / P)^{0}}$ $=A^{G(A / B)^{0}}$ and $G(A / B)^{0}$ is regular. Moreover $D$ is a Galois extension of $P$ and $[D: P]=\operatorname{card}(G(D / P))$. In fact, we see as above for $G(B / P)$ that $G(D / P)$ is finite and contains no nontrivial inner automorphism, and $D^{G(D / P)}$ $=A^{G(A / P)}=P$. Now $D$ is a Galois extension of $B$ and $[D: B]=\operatorname{card}(G(D / B))$, because $B$ is a regular subring of $D$ ( $B$ is a simple ring with minimum condition, $[D: B] \leqq[A: B]<\infty$ and $Z(D, B)=Z(D))$. Next, $B$ is a Galois extension of $P$, since $B$ is a normal extension of $P$ and $G(B / P)$ is finite and contains 
no nontrivial inner automorphism. Therefore $[B: P]=\operatorname{card}(G(B / P))$. By (vi), every element of $G(B / P)$ is the restriction to $B$ of an element of $G(D / P)$. Choose elements $\psi_{1}, \cdots, \psi_{[B: P]}$ in $G(D / P)$ whose restrictions to $B$ make up the group $G(B / P)$. Then the cosets $\psi_{i} G(D / B)$ are distinct, so that $\bigcup_{i=1}^{[B: P]} \psi_{i} G(D / B)$ consists of $[D: B] \cdot[B: P]=[D: P]$ elements of $G(D / P)$. Hence $G(D / P)=\bigcup_{i=1}^{[B: P]} \psi_{i} G(D / B)$. If $\phi \in G(D / B)$ then $\psi_{i} \phi(B)=\psi_{i}(B)=B$. Hence $\psi(B)=B$, for every $\psi \in G(D / P)$, i.e., $B$ is a $D$-normal extension of $P$. This completes the proof of Lemma 5.

Theorem 2. Let $A$ be a Galois extension of $C$, and let $B_{1}, B_{2}$ be regular subrings between $C$ and $A$. Moreover, let us suppose that $B_{1}$ and $B_{2}$ are normal extensions of $B_{1} \cap B_{2}$, and $Z(A)$ is infinite.

I. The following two conditions are equivalent, for $i=1,2$.

$\left(\mathrm{a}_{\mathrm{i}}\right) B_{i}$ is an $A$-normal extension of $B_{1} \cap B_{2}$ and $G\left(B_{i} / B_{1} \cap B_{2}\right)$ contains no nontrivial inner automorphism.

$\left(\mathrm{b}_{\mathrm{i}}\right) Z\left(A, B_{1} \cap B_{2}\right)=Z\left(A, B_{i}\right)$.

Moreover, if one of the conditions is satisfied then $B_{1} \cap B_{2}$ is a regular subring of $A$, and $G\left(B_{i} / B_{1} \cap B_{2}\right)$ is finite.

II. The following three conditions are equivalent:

(a) $\left(a_{1}\right)$ or $\left(a_{2}\right)$,

(b) $\left(b_{1}\right)$ or $\left(b_{2}\right)$,

(c) every compatible pair $\left(\phi_{1}, \phi_{2}\right)$, where $\phi_{i} \in G\left(B_{i}, A / C\right)$, for $i=1,2$, has an extension $\phi \in G(A / C)$.

Proof. I. $\left(\mathrm{b}_{\mathrm{i}}\right) \Rightarrow\left(\mathrm{a}_{\mathrm{i}}\right)$. If $Z\left(A, B_{1} \cap B_{2}\right)=Z\left(A, B_{i}\right)$ then $G\left(B_{i} / B_{1} \cap B_{2}\right)$ contains no nontrivial inner automorphism and $B_{i} \subset A^{G\left(A / B_{1} \cap B_{2}\right)^{0}}$. Hence it follows from Lemma 5 that $B_{i}$ is an $A$-normal extension of $B_{1} \cap B_{2}, B_{1} \cap B_{2}$ is a regular subring of $A$ and $G\left(B_{i} / B_{1} \cap B_{2}\right)$ is finite.

$\left(\mathrm{a}_{\mathrm{i}}\right) \Rightarrow\left(\mathrm{b}_{\mathrm{i}}\right)$. Let $h$ be the map from $G\left(A / B_{1} \cap B_{2}\right)$ into $G\left(B_{i} / B_{1} \cap B_{2}\right)$ defined by $h(\psi)=\psi \mid B_{i}$, for every $\psi \in G\left(A / B_{1} \cap B_{2}\right)$. Then $h$ is a homomorphism. $G\left(B_{i} / B_{1} \cap B_{2}\right)$ contains no nontrivial inner automorphism. Hence, it follows from (iii) that, $\operatorname{card}\left(G\left(B_{i} / B_{1} \cap B_{2}\right)\right) \leqq\left[G\left(B_{i} / C\right): G\left(B_{i} / C\right)^{0}\right] \leqq\left[B_{i}: C\right]<\infty$. Therefore $h\left(G\left(A / B_{1} \cap B_{2}\right)^{0}\right)$ is also finite. But $h\left(G\left(A / B_{1} \cap B_{2}\right)^{0}\right)$ $\approx Z\left(A, B_{1} \cap B_{2}\right)^{*} / Z\left(A, B_{i}\right)^{*}$. Hence there exists a finite set $\left\{d_{1}, \cdots, d_{n}\right\}$ $C Z\left(A, B_{1} \cap B_{2}\right)$ such that $Z\left(A, B_{1} \cap B_{2}\right)^{*}=\bigcup_{j=1}^{n} Z\left(A, B_{i}\right)^{*} d_{j} . Z\left(A, B_{i}\right)$ is a simple ring with minimum condition and $\left[Z\left(A, B_{1} \cap B_{2}\right): Z(A)\right]<\infty$. Hence the assumptions of Lemma 4 are satisfied for $C_{j}=D_{j}=Z\left(A, B_{i}\right), F=Z(A)$, $R=Z\left(A, B_{1} \cap B_{2}\right)$. Thus $Z\left(A, B_{1} \cap B_{2}\right)=Z\left(A, B_{i}\right)$.

II. The implications (b) $\Rightarrow$ (a) and (c) $\Rightarrow$ (b) follow from the first part of this theorem and Theorem 1, respectively. Hence it remains to prove that $\left(\mathrm{a}_{\mathrm{i}}\right)$ implies (c) for $i=1,2$.

Let us suppose that $\left(a_{1}\right)$ holds. Then $G\left(B_{1}, A / B_{1} \cap B_{2}\right)=G\left(B_{1} / B_{1} \cap B_{2}\right)$, since $B_{1}$ is an $A$-normal extension of $B_{1} \cap B_{2}$. Now $G\left(A / B_{2}\right) \mid B_{1}$ is a complete subgroup of $G\left(B_{1} / B_{1} \cap B_{2}\right)$, because $G\left(B_{1} / B_{1} \cap B_{2}\right)$ does not contain any non- 
trivial inner automorphism. Hence $G\left(A / B_{2}\right) \mid B_{1}$ is a regular subgroup of $G\left(B_{1} / B_{1} \cap B_{2}\right)$, since it follows from the first part of the theorem that $G\left(B_{1} / B_{1} \cap B_{2}\right)$ is finite. Moreover $B_{1}^{G\left(A / B_{2}\right) \mid B_{1}}=A^{G\left(A / B_{2}\right)} \cap B_{1}=B_{2} \cap B_{1}$. Hence it follows from (v) that $G\left(A / B_{2}\right) \mid B_{1}=G\left(B_{1} / B_{1} \cap B_{2}\right)$. Therefore $G\left(A / B_{2}\right) \mid B_{1}$ $=G\left(B_{1}, A / B_{1} \cap B_{2}\right)$ and hence, by Lemma 1 , we obtain (c). Similarly, we prove that $\left(a_{2}\right)$ implies (c). Thus (a) implies (c) and this completes the proof of Theorem 2.

Corollary 3. Let $A$ be a Galois extension of $C$ and let $B_{1}, B_{2}$ be regular subrings between $C$ and $A$. Moreover, let us suppose that $B_{1}, B_{2}$ are normal extensions of $B_{1} \cap B_{2}, Z(A)$ is infinite, and $A=\left(B_{1}, B_{2}\right)$.

If every compatible pair $\left(\phi_{1}, \phi_{2}\right)$, where $\phi_{i} \in G\left(B_{i}, A / C\right)$, for $i=1,2$, has an extension $\phi \in G(A / C)$ then $B_{1} \cap B_{2}$ is a regular subring of $A\left[A: B_{1} \cap B_{2}\right]$ $=\left[A: B_{1}\right]\left[A: B_{2}\right],\left[A: B_{1}\right]=\left[B_{2}: B_{1} \cap B_{2}\right]$ and $\left[A: B_{2}\right]=\left[B_{1}: B_{1} \cap B_{2}\right]$.

Proof. Let us suppose that every compatible pair $\left(\phi_{1}, \phi_{2}\right)$, where $\phi_{i} \in G\left(B_{i}, A / C\right)$, for $i=1,2$, has an extension $\phi \in G(A / C)$. Then it follows from Theorem 2 that, either for $i=1$ or else for $i=2, Z\left(A, B_{1} \cap B_{2}\right)=Z\left(A, B_{i}\right)$, $B_{i}$ is an $A$-normal extension of $B_{1} \cap B_{2}$ and $G\left(B_{i} / B_{1} \cap B_{2}\right)$ is finite and does not contain any nontrivial inner automorphism. Let us suppose that this holds for $i=1$. Then $G\left(A / B_{2}\right)$ contains no nontrivial inner automorphism. Indeed $Z\left(A, B_{2}\right) \subset Z\left(A, B_{1} \cap B_{2}\right)=Z\left(A, B_{1}\right)$, and so $Z\left(A, B_{2}\right) \subset Z\left(A, B_{1}\right) \cap Z\left(A, B_{2}\right)$ $=Z\left(A,\left(B_{1}, B_{2}\right)\right)=Z(A, A)=Z(A)$. Let $h$ be the homomorphism of $G\left(A / B_{2}\right)$ into $G\left(B_{1} / B_{1} \cap B_{2}\right)$ defined by $h(\psi)=\psi \mid B_{1}$, for every $\psi \in G\left(A / B_{2}\right)\left(B_{1}\right.$ is an $A$-normal extension of $B_{1} \cap B_{2}$; hence $\psi \mid B_{1} \in G\left(B_{1} / B_{1} \cap B_{2}\right)$ for every $\left.\psi \in G\left(A / B_{2}\right)\right)$. Then $h\left(G\left(A / B_{2}\right)\right)$ is a regular subgroup of $G\left(B_{1} / B_{1} \cap B_{2}\right)$, because $G\left(B_{1} / B_{1} \cap B_{2}\right)$ is finite and does not contain any nontrivial inner automorphism. Moreover $B_{1}^{h\left(G\left(A / B_{2}\right)\right)}=B_{1}^{G\left(A / B_{2}\right) \mid B_{1}}=A^{G\left(A / B_{2}\right)} \cap B_{1}=B_{1} \cap B_{2}$. Hence $h\left(G\left(A / B_{2}\right)\right)=G\left(B_{1} / B_{1} \cap B_{2}\right)$. But $h$ is a monomorphism, since $A=\left(B_{1}, B_{2}\right)$. Therefore, $h$ maps $G\left(A / B_{2}\right)$ isomorphically onto $G\left(B_{1} / B_{1} \cap B_{2}\right)$ and $\operatorname{card}\left(G\left(A / B_{2}\right)\right)=\operatorname{card}\left(G\left(B_{1} / B_{1} \cap B_{2}\right)\right)$. By Theorem 2, $B_{1} \cap B_{2}$ is regular in $A$. Hence $\left[A: B_{1} \cap B_{2}\right],\left[B_{1}: B_{1} \cap B_{2}\right]$ and $\left[B_{2}: B_{1} \cap B_{2}\right]$ are well defined and finite. Moreover, $\left[A: B_{2}\right]=\operatorname{card}\left(G\left(A / B_{2}\right)\right)=\operatorname{card}\left(G\left(B_{1} / B_{1} \cap B_{2}\right)\right)=\left[B_{1}: B_{1} \cap B_{2}\right]$. But $\left[A: B_{1} \cap B_{2}\right]=\left[A: B_{1}\right] \cdot\left[B_{1}: B_{1} \cap B_{2}\right]=\left[A: B_{2}\right]\left[B_{2}: B_{1} \cap B_{2}\right]$. Hence $\left[A: B_{1} \cap B_{2}\right]=\left[A: B_{1}\right]\left[A: B_{2}\right],\left[A: B_{1}\right]=\left[B_{2}: B_{1} \cap B_{2}\right]$ and $\left[A: B_{2}\right]$ $=\left[B_{1}: B_{1} \cap B_{2}\right]$.

3. In the sequel, letters $F, K, L, M, N$ will denote division rings. If $F$ is a division subring of $K$ then the set of all $F$-linear endomorphisms of $K$ will be denoted by $E(K ; F)$.

Let $F \subset N \subset K$ be successive extensions of $F$. Then $E(N, K ; F)$ denotes the set of all $F$-linear mappings of $N$ into $K$. It is easy to see that $G(N, K / F)$ $C E(N, K ; F)$. If $f \in E(N, K ; F), a \in N$ and $b \in K$ then $f \cdot a_{r} \in E(N, K ; F)$ and $b_{r} \cdot f \in E(N, K ; F)$. Hence $E(N, K ; F)$ can be considered as a $(K ; N)$-module.

The following easy result is recorded here for reference: 
(vii) Let $K$ be a finite extension of $F$. Then

(a) $F$ is a regular subring of $K$,

(b) a subgroup $G_{1}$ of $G(K / F)$ is regular if and only if $G_{1}$ is complete. In particular $G(K / F)$ is regular.

(c) $K$ is a Galois extension of $F$ if and only if $K$ is a normal extension of $F$.

The following theorem follows from (vi) and (vii):

(viii) (15) Let $K$ be a finite and normal extension of $F$ and let $N$ be an extension of $F$ such that $N \subset K$. Every isomorphism $\phi \in G(N, K / F)$ has an extension $\psi \in G(K / F)$.

Let $N_{1}, N_{2}$ be two extensions of $F$ and let $K$ be an extension of $N_{1}$ and $N_{2}$. We shall say that $N_{1}$ is linearly disjoint from $N_{2}$ if every subset of $N_{1}$ that is left linearly independent over $N_{1} \cap N_{2}$ is also left linearly independent over $N_{2}$. If $N_{1}$ is linearly disjoint from $N_{2}$, and $N_{2}$ is linearly disjoint from $N_{1}$, then we say that $N_{1}, N_{2}$ are linearly disjoint.

We shall use the following theorems.

(ix) $\left.{ }^{16}\right)$ Let $K$ be a normal and finite extension of $F$. Then

$$
\left(K_{r}, G(K / F)\right)=E(K ; F) .
$$

(x) Let $K$ be a normal and finite extension of $F$ and let $N$ be a subring of $K$ containing $F$. If $\phi, \psi_{1}, \cdots, \psi_{n} \in G(N, K / F)$ and $\phi=\sum_{i=1}^{n} a_{r}^{i} \psi_{i}$, where $a^{i} \in K$, then there exist $j,(1 \leqq j \leqq n)$, and an element $a \in Z(K, F)$ such that $\phi=I_{a-1} \psi_{j}$.

The following proof of $(\mathrm{x})$ is contained in the proof of Theorem 1 [12, p. 162]:

It is easy to see that if $\psi \in G(N, K / F)$ then $K_{r} \cdot \psi$ is a simple $(K ; N)$ module; hence $K_{r} \psi_{1}+\cdots+K_{r} \psi_{n}$ is a completely reducible $(K ; N)$-module and $K_{r} \phi \subset K_{r} \psi_{1}+\cdots+K_{r} \psi_{n}$ implies that there exists $j, 1 \leqq j \leqq n$, such that $K_{r} \phi$ is $(K ; N)$-isomorphic to $K_{r} \psi_{j}$. Let us suppose that $h$ is a $(K ; N)$-isomorphism of $K_{r} \phi$ onto $K_{r} \psi_{j}$ and let $h(\phi)=a_{r} \psi_{j}$ where $a \in K$. Then for any $x \in N$ we have

$$
a_{r} \psi_{j}(x)_{r} \psi_{j}=a_{r} \psi_{j} x_{r}=h(\phi) x_{r}=h\left(\phi x_{r}\right)=h\left(\phi(x)_{r} \cdot \phi\right)=\phi(x)_{r} h(\phi)=\phi(x)_{r} a_{r} \psi_{j}
$$

and therefore $\psi_{j}(x) a=a \phi(x)$. Thus $\phi=a_{l}^{-1} a_{r} \psi_{j}=I_{a^{-1}} \psi_{j}$.

Lemma 6. Let $K$ be a finite and normal extension of $F$ and let $N_{1}, N_{2}$ be two division rings between $F$ and $K$. If $G\left(K / N_{2}\right) \mid N_{1}=G\left(N_{1}, K / N_{1} \cap N_{2}\right)$ then $N_{1}$, $\mathrm{N}_{2}$ are linearly disjoint.

Proof. Let us suppose that $G\left(K / N_{2}\right) \mid N_{1}=G\left(N_{1}, K / N_{1} \cap N_{2}\right)$. It follows from (vii) and Corollary 1 that it suffices to prove that $N_{1}$ is linearly disjoint from $N_{2}$. Let $f$ be any $N_{1} \cap N_{2}$-linear mapping of $N_{1}$ into $K$. By (ix) $f$ can be represented in the form $\sum_{i=1}^{n}\left(a_{t}^{i} \phi_{i}\right) \mid N_{1}$, where $a^{i} \in K$ and $\phi_{i} \in G\left(K / N_{1} \cap N_{2}\right)$,

(15) Cf. Theorem 3, p. 66 in [5].

(16) See [11] or [5]. 
for $i=1,2, \cdots, n$. It follows from our assumption that for every $\phi_{i}, i=1,2$, $\cdots, n$, there exists an automorphism $\psi_{i} \in G\left(K / N_{2}\right)$ such that $\phi_{i}\left|N_{1}=\psi_{i}\right| N_{1}$. Therefore $f=\sum_{i=1}^{n}\left(a_{r}^{i} \psi_{i}\right) \mid N_{1}$. Hence $f$ can be extended to a $N_{2}$-linear mapping $\sum_{i=1}^{n} a_{r}^{i} \psi_{i}$. Thus we have shown that every $\left(N_{1} \cap N_{2}\right)$-linear mapping of $N_{1}$ into $K$ can be extended to an $N_{2}$-linear mapping of $K$ into $K$. Clearly, this implies that $N_{1}$ is linearly disjoint from $N_{2}$.

Leмma 7. Let $K$ be a finite and normal extension of $F$ and let $N_{1}, N_{2}$ be two division rings between $F$ and $K$. If $N_{1}$ is linearly disjoint from $N_{2}$ and $Z\left(K, N_{1} \cap N_{2}\right)=Z\left(K, N_{2}\right)$ then $G\left(N_{1}, K / N_{1} \cap N_{2}\right)=G\left(K / N_{2}\right) \mid N_{1}$.

Proof. Let $\phi \in G\left(N_{1}, K / N_{1} \cap N_{2}\right)$. If $N_{1}$ is linearly disjoint from $N_{2}$ then $\phi$ can be extended to an $N_{2}$-linear mapping $f$ of $K$ into $K$. It follows from (viii) that $f$ can be represented in the form $\sum_{i=1}^{n} a_{r}^{i} \phi_{i}$, where $\phi_{i} \in G\left(K / N_{2}\right)$ and $a^{i} \in K$, for $i=1, \cdots, n$. Hence $\phi=\sum_{i=1}^{n} a_{r}^{i}\left(\phi_{i} \mid N_{1}\right)$. It follows from (x) that there exist an integer $j, 1 \leqq j \leqq n$, and an element $a \in Z\left(K, N_{1} \cap N_{2}\right)$ such that $\phi=I_{a} \cdot\left(\phi_{j} \mid N_{1}\right)=\left(I_{a} \phi_{j}\right) \mid N_{1}$. If $Z\left(K, N_{1} \cap N_{2}\right)=Z\left(K, N_{2}\right)$, we have $I_{a} \in G\left(K / N_{2}\right)$, and hence $I_{a} \phi_{j} \in G\left(K / N_{2}\right)$. Thus $\phi \in G\left(K / N_{2}\right) \mid N_{1}$ and the lemma is proved.

Theorem 3. Let $N_{1}, N_{2}$ be two finite extensions of $F$, and let $K$ be an extension of $N_{1}, N_{2}$, normal and finite over $F$, and suppose that $Z(K)$ is infinite. Every compatible pair $\left(\phi_{1}, \phi_{2}\right)$, where $\phi_{i} \in G\left(N_{i}, K / F\right)$, for $i=1,2$, has an extension $\phi \in G(K / F)$ if and only if $N_{1}, N_{2}$ are linearly disjoint and either $Z\left(K, N_{1} \cap N_{2}\right)=Z\left(K, N_{1}\right)$ or $Z\left(K, N_{1} \cap N_{2}\right)=Z\left(K, N_{2}\right)$.

Proof. If $N_{1}, N_{2}$ are linearly disjoint and (e.g.) $Z\left(K, N_{1} \cap N_{2}\right)=Z\left(K, N_{2}\right)$ then it follows from Lemma 7 that $G\left(N_{1}, K / N_{1} \cap N_{2}\right)=G\left(K / N_{2}\right) \mid N_{1}$. Therefore, by Lemma 1 , every compatible pair $\left(\phi_{1}, \phi_{2}\right)$, where $\phi_{i} \in G\left(N_{i}, K / F\right)$, $i=1,2$, has an extension $\phi \in G(K / F)$.

Conversely, let us suppose that every compatible pair $\left(\phi_{1}, \phi_{2}\right)$, where $\phi_{i} \in G\left(N_{i}, K / F\right)$, for $i=1,2$, has an extension $\phi \in G(K / F)$. Then it follows from Lemmas 1 and 6 that $N_{1}, N_{2}$ are linearly disjoint, and from Theorem 1 and (vii) that either $Z\left(K, N_{1} \cap N_{2}\right)=Z\left(K, N_{1}\right)$ or $Z\left(K, N_{1} \cap N_{2}\right)=Z\left(K, N_{2}\right)$. This completes the proof of the theorem.

Let $N$ and $M$ be two division subrings of $K$. Then we may consider $K$ as an $(N ; M)$-module. This module will be denoted by ${ }_{N} K_{M}$.

Corollary 4. Let $K, M, N$ be finite and normal extensions of $F$ such that $K=(M, N)$. Let $L=M \cap N$ and suppose that $Z(K)$ is infinite. If every compatible pair $\left(\phi_{1}, \phi_{2}\right)$, where $\phi_{1} \in G(M, K / F), \phi_{2} \in G(N, K / F)$, has an extension $\phi \in G(K / F)$ then the map $f:{ }_{M} M_{L} \otimes_{L}{ }_{L} N_{N}$, where $f\left(\sum_{i=1}^{r} x_{i} \otimes y_{i}\right)=\sum_{i=1}^{r} x_{i} y_{i}$, for every $x_{i} \in M, y_{i} \in N$, is an isomorphism of ${ }_{M} M_{L} \otimes_{L}{ }_{L} N_{N}$ onto ${ }_{M} K_{N}$.

Proof. Clearly, $f$ is an $(M ; N)$-homomorphism of ${ }_{M} M_{L} \otimes_{L}{ }_{L} N_{N}$ into ${ }_{M} K_{N}$. Let $b_{1}, \cdots, b_{n}$ be a left $L$-basis of $N$. Then it follows from Theorem 3 that, 
under the assumptions of our corollary, $M$ and $N$ are linearly disjoint. Hence $b_{1}, \cdots, b_{n}$ are left $M$-linearly independent. It follows that $f$ is a monomorphism and that the left dimension of $f\left({ }_{M} M_{L} \otimes_{L}{ }_{L} N_{N}\right)$ over $M$ is equal to $n=[N: L]$. Since $M$ and $N$ are normal over $L$, Corollary 3 and our assumption imply that $[K: M]=[N: L]$. Hence $f$ is an epimorphism, and therefore an isomorphism of ${ }_{M} M_{L} \otimes_{L}{ }_{L} N_{N}$ onto ${ }_{M} K_{N}$.

4. Letters $A, B, B_{1}, B_{2}, C$ will, as before, denote simple rings with minimum condition. Let $C, B$ be subrings of $A$ and let $B$ be between $C$ and $A$. Then $D(B, A / C)$ denotes the set of all derivations of $B$ into $A$ mapping $C$ into (0). Instead of $D(A, A / C)$ we shall write $D(A / C)$. If $a \in A$ then the map $d_{a}$ defined by $d_{a}(x)=a x-x a$, for all $x \in A$, is a derivation. $d_{a}$ is an element of $D(A / C)$ if and only if $a \in Z(A, C)$.

In the sequel we shall use the following theorem:

(xi) ( $\left.{ }^{17}\right)$ Let $A$ be a Galois extension of $C$ and $B$ be between $C$ and $A$. If $d \in D(B, A / C)$ then there exists $a \in Z(A, C)$ such that $d_{a} \mid B=d$. In particular every derivation $d \in D(A / C)$ is inner.

Let $C, B_{1}, B_{2}$ be subrings of $A$ and let $B_{1}, B_{2}$ be between $C$ and $A$. If $d_{i} \in D\left(B_{i}, A / C\right)$, for $i=1,2$, then an element $d \in D(A / C)$ is called an extension of the pair $\left(d_{1}, d_{2}\right)$ if $d \mid B_{i}=d_{i}$, for $i=1,2$. The pair $\left(d_{1}, d_{2}\right)$ is called compatible if $d_{1}\left|B_{1} \cap B_{2}=d_{2}\right| B_{1} \cap B_{2}$.

Lemma 8. Let $A$ be a Galois extension of $C$ and let $B_{1}, B_{2}$ be between $C$ and $A$. Then the following three conditions are equivalent:

(a) every compatible pair $\left(d_{1}, d_{2}\right)$, where $d_{i} \in D\left(B_{i}, A / C\right)$, for $i=1,2$ has an extension $d \in D(A / C)$.

(b) $D\left(A / B_{1} \cap B_{2}\right)=D\left(A / B_{1}\right)+D\left(A / B_{2}\right)$.

(c) $Z\left(A, B_{1} \cap B_{2}\right)=Z\left(A, B_{1}\right)+Z\left(A, B_{2}\right)$.

Proof. (a) $\Rightarrow$ (b). Let $d \in D\left(A / B_{1} \cap B_{2}\right)$. It follows from (a) that there exists a derivation $d_{1} \in D(A / C)$ such that $d_{1}\left|B_{2}=d\right| B_{2}$ and $d_{1} \mid B_{1}$ is the zero derivation of $B_{1}$. Now $d=d_{1}+\left(d-d_{1}\right), d-d_{1} \in D\left(A / B_{2}\right)$ and $d_{1} \in D\left(A / B_{1}\right)$. Thus $d \in D\left(A / B_{1}\right)+D\left(A / B_{2}\right)$.

(b) $\Rightarrow$ (c). Let $c \in Z\left(A, B_{1} \cap B_{2}\right)$. Then $d_{c} \in D\left(A / B_{1} \cap B_{2}\right)$. Hence $d_{c}$ can be represented in the form $d^{\prime}+d^{\prime \prime}$ where $d^{\prime} \in D\left(A / B_{1}\right)$ and $d^{\prime \prime} \in D\left(A / B_{2}\right)$. It follows from (xi) that $d^{\prime}$ and $d^{\prime \prime}$ are inner. Hence there exist elements $a$ and $b$ such that $d_{c}=d_{a}+d_{b}, d_{a}=d^{\prime} \in D\left(A / B_{1}\right)$ and $d_{b}=d^{\prime \prime} \in D\left(A / B_{2}\right)$. Therefore $a \in Z\left(A, B_{1}\right), b \in Z\left(A, B_{2}\right)$ and $c=a+b+z$ where $z \in Z(A)$. Thus $c \in Z\left(A, B_{1}\right)$ $+Z\left(A, B_{2}\right)$.

(c) $\Rightarrow(\mathrm{a})$. Let us take $\left(d_{1}, d_{2}\right)$ as in (a). It follows from (xi) that there exists $a \in Z(A, C)$ and $a_{1}, a_{2} \in Z\left(A, B_{1} \cap B_{2}\right)$ such that $d\left|B_{1} \cap B_{2}=d_{a}\right| B_{1} \cap B_{2}$ and $d_{a_{i}} \mid B_{i}=d_{i}-d_{a}$, for $i=1,2 . a_{1}$ and $a_{2}$ can be represented in the form $a_{1}=a_{1}^{\prime}$ $+a_{1}^{\prime \prime}, a_{2}=a_{2}^{\prime}+a_{2}^{\prime \prime}$, where $a_{1}^{\prime}, a_{2}^{\prime} \in Z\left(A, B_{1}\right)$ and $a_{1}^{\prime \prime}, a_{2}^{\prime \prime} \in Z\left(A, B_{2}\right)$. Let $b=a_{1}^{\prime \prime}+a_{2}^{\prime}$. Then

(17) (xi) is a particular case of Theorem 1, p. 151 in [12]. 


$$
\begin{aligned}
& d_{b}\left|B_{1}=d_{a^{\prime \prime}{ }_{1}}\right| B_{1}=d_{a_{1}} \mid B_{1}=d_{1}-\left(d_{a} \mid B_{1}\right), \\
& d_{b}\left|B_{2}=d_{a_{2}^{\prime}}\right| B_{2}=d_{a_{2}} \mid B_{2}=d_{2}-\left(d_{a} \mid B_{2}\right) .
\end{aligned}
$$

Therefore $d_{b}+d_{a}$ is an extension of $\left(d_{1}, d_{2}\right)$ to a derivation of $A$.

LeмmA 9. Let $R$ be an arbitrary ring with 1 and let $B_{1}, B_{2}, C$ be simple subrings of $R$ satisfying the minimum condition and containing 1. If $B_{1} \cap B_{2} \subset C$, $[R: C]<\infty$ and $R=B_{1}+B_{2}$ then either $R=B_{1}$ or $R=B_{2}$.

Proof. There exists a division ring $L$ such that $[C: L]<\infty$, and we may suppose that $\left[B_{1}: L\right] \geqq\left[B_{2}: L\right]$. Then we have $[R: L] \leqq\left[B_{1}: L\right]+\left[B_{2}: L\right]$ $-1<2\left[B_{1}: L\right]$, since $R=B_{1}+B_{2}$. On the other hand, $[R: L]=\left[R: B_{1}\right]\left[B_{1}: L\right]$. Therefore $\left[R: B_{1}\right]<2$. Hence $\left[R: B_{1}\right]=1$ and so $R=B_{1}$.

TheOREM 4. Let $A$ be a Galois extension of $C$ and let $B_{1}, B_{2}$ be two regular subrings of $A$ containing $C$. Then the following three conditions are equivalent:

(a) every compatible pair $\left(d_{1}, d_{2}\right)$, where $d_{i} \in D\left(B_{i}, A / C\right)$, for $i=1,2$, has an extension $d \in D(A / C)$.

(b) $D\left(B_{1}, A / B_{1} \cap B_{2}\right)=0$ or $D\left(B_{2}, A / B_{1} \cap B_{2}\right)=0$.

(c) $Z\left(A, B_{1} \cap B_{2}\right)=Z\left(A, B_{1}\right)$ or $Z\left(A, B_{1} \cap B_{2}\right)=Z\left(A, B_{2}\right)$.

Proof. It is easy to see that (b) $\Rightarrow(c)$. By (xi), (c) $\Rightarrow(b)$. Hence it suffices to prove that $(\mathrm{a}) \Rightarrow(\mathrm{c})$ and $(\mathrm{b}) \Rightarrow(\mathrm{a})$.

$(\mathrm{a}) \Rightarrow(\mathrm{c})$. If every compatible pair $\left(d_{1}, d_{2}\right)$, where $d_{i} \in D\left(B_{i}, A / C\right)$, for $i=1,2$, has an extension $d \in D(A / C)$ then it follows from Lemma 8 that $Z\left(A, B_{1}\right)+Z\left(A, B_{2}\right)=Z\left(A, B_{1} \cap B_{2}\right)$. Next, $Z\left(A, B_{1}\right)$ and $Z\left(A, B_{2}\right)$ are simple rings with minimum condition, $Z\left(A, B_{1}\right) \cap Z\left(A, B_{2}\right) \subset Z(A)$ and

$$
\left[Z\left(A, B_{1} \cap B_{2}\right): Z(A)\right] \leqq[Z(A, C): Z(A)]<\infty .
$$

Therefore, it follows from Lemma 9 that either $Z\left(A, B_{1} \cap B_{2}\right)=Z\left(A, B_{1}\right)$ or $Z\left(A, B_{1} \cap B_{2}\right)=Z\left(A, B_{2}\right)$.

(b) $\Rightarrow$ (a). Let us suppose that $D\left(B_{1}, A / B_{1} \cap B_{2}\right)=0$ and let $\left(d_{1}, d_{2}\right)$ be a pair as in (a). It follows from (xi) that there exists a derivation $d \in D(A / C)$ such that $d \mid B_{2}=d_{2}$. Then $\left(d \mid B_{1}\right)-d_{1} \in D\left(B_{1}, A / B_{1} \cap B_{2}\right)$. Hence $\left(d \mid B_{1}\right)-d_{1}$ $=0$, and $d$ is an extension of the pair $\left(d_{1}, d_{2}\right)$.

Corollary 5. Let $A, B_{1}, B_{2}, C$ be as in Theorem 4 and let $Z(A)$ be infinite. If every compatible pair $\left(\phi_{1}, \phi_{2}\right)$, where $\phi_{i} \in G\left(B_{i}, A / C\right)$, for $i=1,2$, has an extension $\phi \in G(A / C)$ then every compatible pair $\left(d_{1}, d_{2}\right)$, where $d_{i} \in D\left(B_{i}, A / C\right)$, for $i=1,2$, has an extension $d \in D(A / C)$.

The foregoing corollary is an immediate consequence of Theorem 1 and Theorem 4.

Corollary 6. Let $A, B_{1}, B_{2}, C$ be as in Corollary 5 and let $B_{1}, B_{2}$ be normal extensions of $B_{1} \cap B_{2}$. Every compatible pair $\left(\phi_{1}, \phi_{2}\right)$, where $\phi_{i} \in G\left(B_{i}, A / C\right)$, for 
$i=1,2$, has an extension $\phi \in G(A / C)$ if and only if every compatible pair $\left(d_{1}, d_{2}\right)$, where $d_{i} \in D\left(B_{i}, A / C\right)$, for $i=1,2$, has an extension $d \in D(A / C)$.

The above corollary is a consequence of Corollary 5, Theorem 4, and Theorem 2.

5. In this part we shall prove the following two theorems, where $A, B, C$ are simple rings with minimum condition.

Theorem 5. Let $C$ be a regular subring of $A$, let $B$ be between $C$ and $A$ and suppose that $Z(A)$ is infinite. If $\phi(B)=B$ for every $\phi \in G(A / C)^{\circ}$ then either $B \subset A^{G(A / C)^{0}}$ or $Z(A, C) \subset B$.

Theorem 6. Let $A$ be a Galois extension of $C$, let $B$ be a subring between $C$ and $A$, and suppose that $Z(A)$ is infinite. Then $B$ is an $A$-normal extension of $C$ if and only if $B$ is a normal extension of $C$ and either $B \subset A^{G(A / C)^{\circ}}$ or $Z(A, C)$ $C B$.

As before, $A, B, C$ will stand for simple rings with minimum condition.

Lemma 10. Let $C$ be a subring of $A$, and let $B$ be between $C$ and $A$. Then $[Z(A, C): Z(B)]<\infty$. Moreover, if $Z(A)$ is infinite then $Z(B)$ is also infinite.

Proof. It follows from (iii) that $[Z(A, C): Z(A)]<\infty$ and $[Z(A, B): Z(B)]$ $\leqq[A: B] \leqq[A: C]<\infty$. On the other hand, $Z(A, B) \supset Z(A)$. Hence $[Z(A, C): Z(B)]<\infty$. Thus the first part of the lemma is proved. Now if $Z(B)$ is finite then $Z(A, C)$ is finite and so $Z(A)$ is finite. This completes the proof of the lemma.

Let $C \subset B \subset A$ be successive extensions of $C$. Then $H(A / C, B)$ denotes the subgroup of $G(A / C)$ composed of all elements $I_{a}$, where $a \in Z(A, B)$ * $\cdot Z(B, C)^{*}$. Let $G(A / C, B)$ denote the subgroup of $G(A / C)$ consisting of the elements of $G(A / C)$ that map $B$ onto itself. Clearly, $H(A / C, B)$ is a normal subgroup of $G(A / C, B)$.

Lemma 11. Let $A$ be a finite extension of $C$ and let $B$ be between $C$ and $A$. Then

$$
[G(A / C, B): H(A / C, B)]=\left[G(A / B): G(A / B)^{0}\right] \cdot\left[G(A / C, B) \mid B: G(B / C)^{0}\right] .
$$

Proof. Let $\left\{\psi_{1}, \cdots, \psi_{m}\right\}$ be a set of representatives of the cosets of $G(A / B)^{\circ}$ in $G(A / B)$ and let $\left\{\phi_{1}, \cdots, \phi_{n}\right\}$ be a subset of $G(A / C)$ such that $\left\{\phi_{1}\left|B, \cdots, \phi_{n}\right| B\right\}$ is a set of representatives of the cosets of $G(B / C)^{0}$ in $G(A / C, B) \mid B$. (It follows from (iii) that the sets are finite.) Let $\phi \in G(A / C, B)$. Then there exists $a \in Z(B, C)^{*}$ and an integer $i, 1 \leqq i \leqq n$, such that $\phi \mid B$ $=\left(\phi_{i} I_{a}\right) \mid B$. Thus $\phi\left(\phi_{i} I_{a}\right)^{-1} \in G(A / B)$ and hence there exist $b \in Z(A, B)^{*}$ and an integer $j, 1 \leqq j \leqq m$, such that $\phi\left(\phi_{i} I_{a}\right)^{-1}=\psi_{j} I_{b}$. Therefore $\phi=\psi_{j} I_{b} \phi_{i} I_{a}$ $=\psi_{j} \phi_{i} I_{\phi_{i}{ }^{-1}(b)} I_{a}$. But $\phi_{i}^{-1}(B)=B$ and so $\phi_{i}^{-1}\left(Z(A, B)^{*}\right)=Z(A, B)^{*}$. Hence we have proved that every element $\phi \in G(A / C, B)$ can be represented in the 
form $\psi_{j} \phi_{i} I_{c}$, where $1 \leqq j \leqq m, 1 \leqq i \leqq n$ and $c \in Z(A, B)^{*} \cdot Z(B, C)^{*}$. Thus $G(A / C, B)=\bigcup_{i, j} \psi_{j} \phi_{i} H(A / C, B)$. But it is easy to see that $\psi_{j_{1}} \phi_{i_{1}}$ and $\psi_{j_{2}} \phi_{i_{2}}$ belong to the same coset of $H(A / C, B)$ in $G(A / C, B)$ if and only if $j_{1}=j_{2}$ and $i_{1}=i_{2}$. Hence

$$
[G(A / C, B): H(A / C, B)]=m \cdot n=\left[G(A / B): G(A / B)^{0}\right]\left[G(A / C, B) B: G(B / C)^{0}\right] \text {. }
$$

LEMMA 12. Let $R$ be a ring with the identity 1 , let $F$ be an infinite subfield of $R$ containing 1 and suppose that $[R: F]<\infty$. Let $P$ be a subring between $F$ and R. If $\left[R^{*}: P^{*}\right]<\infty$ then $R=P$.

Proof. We shall consider $R$ and $P$ as left $F$-vector spaces. Let us suppose that $\left[R^{*}: P^{*}\right]<\infty$. Then there exists a finite subset $\left\{a_{i}\right\}_{i=1, \cdots, n}$ of $R^{*}$ such that $R^{*}=\bigcup_{i=1}^{n} P^{*} a_{i}$. Therefore $\left(R^{*}\right)^{-}=\bigcup_{i=1}^{n}\left(P^{*}\right)^{-} a_{i}$. But it follows from Lemma 2 that $\left(R^{*}\right)^{-}=R$ and $\left(P^{*}\right)^{-}=P$. Hence $R=\bigcup_{i=1}^{n} P a_{i}$ and so $R=P a_{j}$ for some $j, 1 \leqq j \leqq n$. Therefore $P=R a_{j}^{-1}=R$ and the proof of the lemma is finished.

REMARKs. The following generalization of the lemma is still true:

Let $R$ be a ring with the identity 1 , let $F$ be an infinite subfield of $R$ containing 1 and suppose that $[R: F]<\infty$. Let $P$ be a subring of $R$. If $\left[R^{*}: P^{*}\right]$ $<\infty$ then $R=P$.

On the other hand the lemma does not remain true if we omit the assumption $[R: F]<\infty$, even in the case where $R$ is a commutative ring.

One can also prove the following theorem:

Let $R$ be an infinite simple ring with minimum condition and let $P$ be a subring of $R$. If $\left[R^{*}: P^{*}\right]<\infty$ then $R=P$.

Proof of Theorem 5. Let us suppose that $B$ is carried into itself by every inner automorphism belonging to $G(A / C)$. Then $Z(A, B)$ and $Z(B, C)$ are carried into themselves by every inner automorphism of $Z(A, C)$. Moreover, $G(A / C)^{0}$ $C G(A / C, B)$ and hence it follows from Lemma 12 that $H(A / C, B)$ is of finite index in $G(A / C)^{0}$. Therefore $\left[Z(A, C)^{*}: Z(A, B)^{*} \cdot Z(B, C)^{*}\right]<\infty$. Furthermore $Z(A, B) \supset Z(B)$ and $Z(B, C) \supset Z(B)$ and it follows from Lemma 11 that $[Z(A, C): Z(B)]<\infty$ and that $Z(B)$ is infinite. Now, $Z(A, B)$ and $Z(B, C)$ are semisimple rings with minimum condition. Indeed, $[Z(A, B): Z(B)]<\infty$ and $[Z(B, C): Z(B)]<\infty$ and hence $Z(A, B), Z(B, C)$ satisfy the minimum condition. Moreover, if $R$ is the radical of $Z(A, B)$ then $a R a^{-1} \subset R$ for every $a \in Z(A, C)^{*}$ (since $a Z(A, B) a^{-1} \subset Z(A, B)$ for every $a \in Z(A, C)^{*}$ ). Hence $Z(A, C) R \subset R Z(A, C)$ because every element from $Z(A, C)$ can be represented as a sum of units. Therefore $Z(A, C) R Z(A, C)$ is nilpotent and hence 0 . Thus $R=0$. Similarly, the radical of $Z(B, C)$ is trivial. Now, if $a \in Z(A, B)$, $b \in Z(B, C)$ then $a b=b a$ and it follows from Lemma 12 that $(Z(A, B), Z(B, C))$ $=Z(A, C)$. Therefore $Z(B) \subset Z(Z(A, C)), Z(Z(A, B)) \subset Z(Z(A, C))$ and $Z(Z(B, C)) \subset Z(Z(A, C))$. But $Z(Z(A, C))$ is a field. Hence $Z(A, B)$ and $Z(B, C)$ are simple rings with minimum condition. Therefore it follows from Lemma 4 that either $Z(A, C)=Z(A, B)$ are $Z(A, C)=Z(B, C)$. If $Z(A, C)$ 
$=Z(A, B)$ then $B \subset A^{G(\boldsymbol{A} / C)^{0}}$. If $Z(A, C)=Z(B, C)$ then $Z(A, C) \subset B$. Therefore the theorem is proved $\left({ }^{18}\right)$.

Corollary 7. Let $C$ be a regular subring of $A$, let $B$ be between $C$ and $A$ and suppose that $Z(A)$ is infinite. If $B$ is carried into itself by every inner automorphism belonging to $G(A / C)$ then $B$ is a regular subring of $A$.

Proof. It follows from Theorem 5 that if $B$ is carried into itself by every inner automorphism belonging to $G(A / C)$ then either $B \subset A^{G(\boldsymbol{A} / C)^{\circ}}$ or $Z(A, C) \subset B$. Suppose that $B \subset A^{G(A / C)^{\circ}}$. Then $Z(A, C)=Z(A, B)$. Therefore $Z(A, B)$ is a simple ring. Moreover $[A: B] \leqq[A: C]<\infty$. Hence $B$ is a regular subring of $A$. Now, let us suppose that $Z(A, C) \subset B$. Then $Z(A, B)=Z(B)$. Therefore $Z(A, B)$ is a field. But, as before, $[A: B] \leqq[A: C]<\infty$. Hence $B$ is a regular subring of $A$. This completes the proof of the corollary.

Proof of Theorem 6. If $B$ is a $A$-normal extension of $C$ then $B$ is a normal extension of $C$ and, by Theorem 5 , either $B \subset A^{G(A / C)^{\circ}}$ or $Z(A, C) \subset B$.

Let $B$ be a normal extension of $C$ and $B C A^{G(A / C)^{\circ}}$. Then $B$ is a regular subring of $A$, since $Z(A, B)=Z(A, C)$. Hence, it follows from Lemma 5 , that $B$ is an $A$-normal extension of $C$.

Now, let $Z(A, C) \subset B$. Then $H(A / C, B)=G(A / C)^{0}$ and, by Lemma 11, $\left[G(A / C, B): G(A / C)^{0}\right]=\left[G(A / B): G(A / B)^{0}\right]\left[G(A / C, B) \mid B: G(B / C)^{0}\right]$.

On the other hand $B$ is a Galois extension of $C$ because $Z(A, C)=Z(B, C)$, $\left[G(B / C): G(B / C)^{0}\right] \leqq[B: C]<\infty$ and $B$ is a normal extension of $C$. Next $B$ is a regular subring of $A$ since $Z(A, B)=Z(B)$. Hence $G(A / C, B) \mid B=G(B, C)$ and

$$
\begin{aligned}
& {\left[G(A / C): G(A / C)^{0}\right]=\frac{[A: C]}{[Z(A, C): Z(A)]}=\frac{[A: B][B: C]}{[Z(A, C): Z(A)]}} \\
& \quad=\frac{\left[G(A / B): G(A / B)^{0}\right][Z(A, B): Z(A)]\left[G(B / C): G(B / C)^{0}\right][Z(B, C): Z(B)]}{[Z(A, C): Z(A)]} \\
& \quad=\frac{[Z(B): Z(A)][Z(A, C): Z(B)]}{[Z(A, C): Z(A)]}\left[G(A / B): G(A / B)^{0}\right]\left[G(B / C): G(B / C)^{0}\right] \\
& \quad=\left[G(A / B): G(A / B)^{0}\right]\left[G(B / C): G(B / C)^{0}\right] .
\end{aligned}
$$

Therefore $G(A / C)=G(A / C, B)$. Hence $B$ is an $A$-normal extension of $C$. The proof is complete.

Corollary 8. Let $A$ be a Galois extension of $C$, let $G(A / C)=G(A / C)^{0}$ and suppose that $Z(A)$ is infinite. If $B$ is an $A$-normal extension of $C$ then either $B=A$ or $B=C$.

The above corollary is an immediate consequence of Theorem 6 .

(18) One can get a somewhat shorter proof of the theorem applying Theorem 1, p. 121 in [6] (Cf. Theorem 3, p. 863 in [2]) or Satz 3, p. 186 in [13] (Cf. also Theorem 3, p. 191 in [10]). 


\section{REFERENCES}

1. S. A. Amitsur, Invariant submodules of simple rings, Proc. Amer. Math. Soc. vol. 7 (1956) pp. 987-989.

2. W. E. Baxter, Lie simplicity of a special class of associative rings, Proc. Amer. Math. Soc. vol. 7 (1957) pp. 855-863.

3. N. Bourbaki, Algèbre, Livre II, Chapitre 8, Paris, Hermann, 1958.

4. R. Brauer, On a theorem of H. Cartan, Bull. Amer. Math. Soc. vol. 55 (1949) pp. 619620.

5. H. Cartan, Théorie de Galois pour les corps non commutatifs, Ann. Sci. Ecole Norm. Sup. vol. 64 (1947) pp. 59-77.

6. A. Hattori, On invariant subrings, Jap. J. Math. vol. 21 (1951) pp. 121-130.

7. G. P. Hochschild, Double vector spaces over division rings, Amer. J. Math. vol. 71 (1949) pp. $443-460$.

8. — Automorphisms of simple algebras, Trans. Amer. Math. Soc. vol. 69 (1950) pp. 292-301. 537.

9. L. K. Hua, Some properties of sfield, Proc. Nat. Acad. Sci. U.S.A. vol. 35 (1949) pp. 533-

10. - A note on the total matrix ring over a non-commutative field, Ann. Soc. Polon. Math. vol. 25 (1952) pp. 188-198.

11. N. Jacobson, $A$ note on division rings, Amer. J. Math. vol. 69 (1947) pp. 27-36.

12. - Structure of rings, Amer. Math. Soc. Colloquium Publications, vol. 37, Providence, 1956.

13. F. Kasch, Invariante Untermoduln des Endomorphismenrings eines Vektorraums, Arch. Math. vol. 4 (1953) pp. 182-190.

14. T. Nakayama, Galois theory of simple rings, Trans. Amer. Math. Soc. vol. 73 (1952) pp. $276-292$.

15. A. Rosenberg, The Cartan-Brauer-Hua theorem for matrix and local matrix rings, Proc. Amer. Math. Soc. vol. 7 (1956) pp. 891-898.

UNiversity of CaLifornia, Berkeley, California

Polisir Acadimy of Sciencis, WARSAW, Poland 